\title{
Constraining the nature of dark energy using the SKA
}

\author{
Angel Torres-Rodriguez and Catherine Cress
}

\author{
Astrophysics and Cosmology Research Unit, University of KwaZulu-Natal, Science Block, \\ Pietermaritzburg, 3209, South Africa \\ email: 204523926@ukzn.ac.za, cressc@ukzn.ac.za
}

\begin{abstract}
Current models for dark energy include a cosmological constant and scalar field models such as quintessence and $k$-essence. By measuring the dark energy sound speed, $c_{\text {eff }}$, and its equation of state parameter, $w$, one can distinguish between these. Here we investigate the possibility of measuring $c_{\text {eff }}$ and $w$ using combined observations from the SKA and CMB experiments. We present theoretical predictions for the cross-power spectrum of ISW fluctuations in the CMB and the expected field of HI galaxies detectable with the SKA.
\end{abstract}

Keywords. Cosmology: CMB, Large-Scale Structure, Dark Energy - Radio Astronomy: HI surveys, SKA.

\section{Introduction}

The Integrated Sachs-Wolfe (ISW) effect arises when CMB photons streaming across the Universe interact with the time evolving gravitational potential wells associated with the foreground large-scale structure. This interaction imprints a secondary anisotropy on the primordial CMB spectrum which can be used to probe the nature of the dark energy driving the accelerated expansion of the Universe.

A common method for observing this effect is through cross-correlation of the CMB spectrum with a tracer of matter at low redshift. In Fourier space, the amplitude of this correlation is given by (see for example Nolta et al. (2004)):

$$
C_{l}^{N T}=4 \pi \int_{0}^{\infty} \frac{d k}{k}\left\langle\frac{\delta N}{N_{0}}(k) \frac{\delta T}{T_{0}}\left(k^{\prime}\right)\right\rangle=4 \pi \int_{0}^{\infty} \frac{d k}{k} f_{l}^{N}(k) f_{l}^{T}(k) \Delta_{m}^{2}(k),
$$

where $\delta N / N_{0}$ and $\delta T / T_{0}$ give the fluctuations in the galaxy numbers and CMB temperature. The filter functions $f_{l}^{N}(k)$ and $f_{l}^{T}(k)$ depend on the linear bias parameter in the HI galaxies $\left(b_{H I}\right)$, the redshift distribution of $\mathrm{HI}$ sources and the suppression of matter fluctuations $(g(z))$. They are expressed as integrals over the comoving distance $(d)$.

The dark energy equation of state parameter, $w$, affects both $d$ and the amplitude of matter fluctuations controlled by $g(z)$. The speed of sound of the dark energy, $c_{e f f}$, has an effect on the evolution of these fluctuations and determines the dark energy clustering properties below the Jeans scale. This information is encoded in the matter power spectrum $\Delta_{m}^{2}(k)$ (see $\mathrm{Hu} 2002$ ).

In order to simulate the HI galaxy distribution that may be available with SKA observations, we follow the analysis of Abdalla \& Rawlings (2005). We particularly focus on their most realistic model (Model $C$ ) which includes HI depletion due to star formation. The total HI density is evaluated from damped-Ly $\alpha$ results. We also assume a linear bias of $b_{H I}=1$. 


\section{ISW Signal and Prospects For Detection}

The cross-correlation signal is presented in figure 1. As $w$ becomes less negative, the signal is increasingly more sensitive to different models of sound speed.

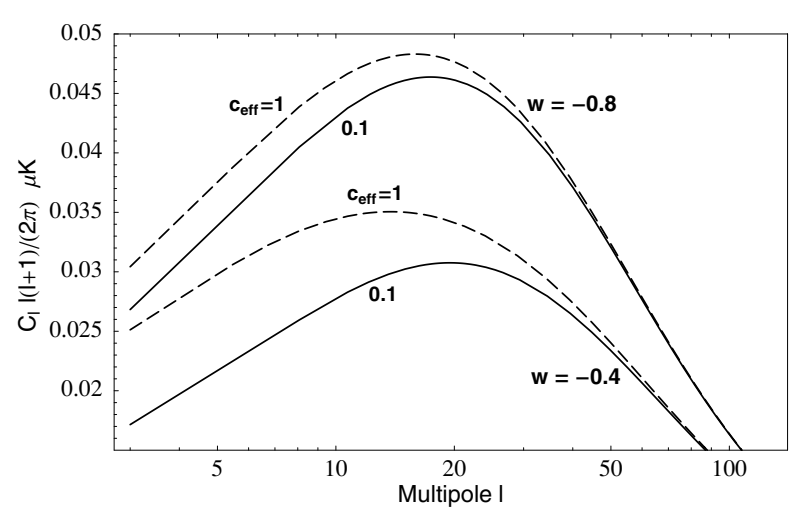

Figure 1. Cross--correlation signal for different values of $w$ and DE sound speed.

In the following figures we provide a measure of the Signal-to-Noise ratio that could be achieved with a SKA-like survey. We follow the approach proposed by Afshordi (2004), dividing the redshift space into a series of (independent) shells of width $\delta z$ (e.g. 0.1).
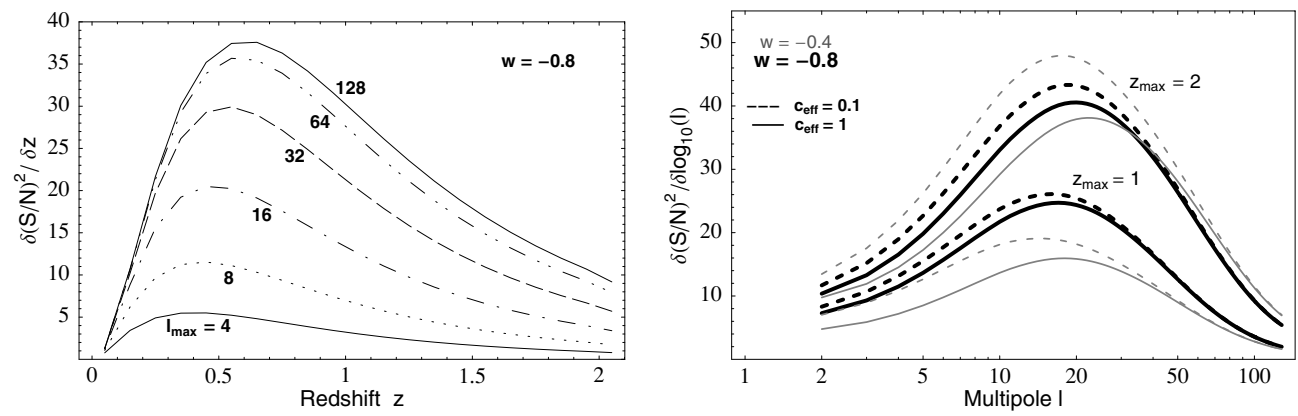

Figure 2. Differential $(S / N)^{2}$ as a function of redshift shell and scale. $z_{\max }$ gives the maximum redshift of the HI survey.

The largest contribution to the signal comes from the redshift range $z \sim 0.3-1.5$ (as expected for the DE domination era) and mainly due to multipoles around $l \sim 20$. Figure 2 indicates that useful constraints on $w$ and $c_{\text {eff }}$ may be achieved from a SKA HI survey.

\section{Acknowledgements}

I would like to acknowledge the grant received from the International Union of Pure and Applied Physics (IUPAP) which supported my attendance to the IAU Symposium 232 held in Cape Town in November 2005.

\section{References}

Abdalla, F.B. \& Rawlings, S. 2005, MNRAS 360, 27

Afshordi, N. 2004, Phys. Rev. D 70, 083536

Hu, W. 2002, Phys. Rev. D 65, 023003

Nolta, M.R., et al. 2004, ApJ 608, 10 